Preprint typeset in JHEP style - HYPER VERSION

\title{
Effects of the galactic magnetic field upon large scale anisotropies of extragalactic Cosmic Rays
}

\author{
D. Harari, S. Mollerach and E. Roulet \\ CONICET, Centro Atómico Bariloche, Bustillo 9500, 8400 Bariloche, Argentina \\ E-mail: harari@cab.cnea.gov.ar,mollerach@gmail.com,eroulet@gmail.com
}

\begin{abstract}
The large scale pattern in the arrival directions of extragalactic cosmic rays that reach the Earth is different from that of the flux arriving to the halo of the Galaxy as a result of the propagation through the galactic magnetic field. Two different effects are relevant in this process: deflections of trajectories and (de)acceleration by the electric field component due to the galactic rotation. The deflection of the cosmic ray trajectories makes the flux intensity arriving to the halo from some direction to appear reaching the Earth from another direction. This applies to any intrinsic anisotropy in the extragalactic distribution or, even in the absence of intrinsic anisotropies, to the dipolar Compton-Getting anisotropy induced when the observer is moving with respect to the cosmic rays rest frame. For an observer moving with the solar system, cosmic rays traveling through far away regions of the Galaxy also experience an electric force coming from the relative motion (due to the rotation of the Galaxy) of the local system in which the field can be considered as being purely magnetic. This produces small changes in the particles momentum that can originate large scale anisotropies even for an isotropic extragalactic flux.
\end{abstract}

KEYWORDS: ultra high energy cosmic rays, galactic magnetics fields. 


\section{Contents}

1. Introduction 1

2. Galactic magnetic field effects on the extragalactic flux 2

3. Quantitative results in a BSS-S model

4. Conclusions 9

\section{Introduction}

The Compton-Getting effect is a dipolar anisotropy due to the Doppler shift of the particle spectrum when the observer is moving with respect to the cosmic rays $(\mathrm{CR})$ rest frame [1]. Interpretation of large scale anisotropy observations requires a knowledge of the contribution due to the Compton-Getting effect. The amplitude $\Delta$ of the resulting dipole depends on the velocity of the observer $v$ and on the cosmic ray spectral index $\gamma$, through $\Delta=(2+\gamma) v / c$. The effect has been observed at the solar daily frequency by several experiments in the $0.1-100 \mathrm{TeV}$ energy range. At this frequency the motion of the Earth around the Sun with a velocity of $\sim 29.8 \mathrm{~km} / \mathrm{s}$ gives rise to a dipolar type anisotropy of amplitude $\Delta \sim 5 \times 10^{-4}$ and maximum at a phase $\phi \sim 6$ hs in local solar time, in good agreement with the observations [2, 3]. At the sidereal daily frequency the computation of the expected Compton-Getting dipolar anisotropy is more uncertain as the rest frame of the local cosmic rays is not known. At relatively low energies the cosmic rays are mostly of Galactic origin and are trapped for a long time in the galactic magnetic field. The first harmonic modulation in right ascension (which is equivalent to the sidereal time) for energies up to a few hundred $\mathrm{TeV}$, measured by different experiments to be $\sim$ few $\times 10^{-4}$, indicates that cosmic rays corotate with the local environment around the Galactic center [4, 5, 6, 7]. If the cosmic rays rest frame were instead linked to an inertial frame fixed to the center of the Galaxy, the solar system velocity of $\sim 220 \mathrm{~km} / \mathrm{s}$ around the galactic center would lead to a much larger $\left(\Delta \sim\right.$ few $\left.\times 10^{-3}\right)$ dipolar anisotropy.

It has been pointed out by Kachelriess and Serpico [8] that the Compton-Getting effect could also induce an observable anisotropy of extragalactic cosmic rays. At the highest energies, cosmic rays are not expected to be confined by the Galactic magnetic field and it is believed that they are mostly of extragalactic origin. The energy at which the transition from the galactic to the extragalactic components occurs is still an open question. According to some models, the ankle in the spectrum at $\sim 4 \mathrm{EeV}$ (where $1 \mathrm{EeV}$ $\equiv 10^{18} \mathrm{eV}$ ) is due to a transition from a steep galactic spectrum to a flatter extragalactic one [9], while another model explains the ankle as a result of the dip produced by $e^{+} e^{-}$ 
pair production by extragalactic protons interacting with the cosmic microwave background $(\mathrm{CMB})[10]$. In the later case, the galactic to extragalactic transition would take place at energies below $1 \mathrm{EeV}$.

The large scale distribution observed at Earth of extragalactic cosmic rays should be affected by the Compton-Getting effect. For energies below the Greisen Zatzepin Kuz'min (GZK) threshold of $\sim 60 \mathrm{EeV}$ they can come from cosmological distances. A reasonable assumption is that the cosmic ray flux at these energies should look isotropic in the CMB rest frame. The observed dipole in the CMB temperature allows to deduce the solar system velocity with respect to the $\mathrm{CMB}$ rest frame, that is $v=371 \pm 1 \mathrm{~km} / \mathrm{s}$ in the $(b, l)=\left(48.4^{\circ}, 264.4^{\circ}\right)$ direction in galactic coordinates [11. This motion would induce a dipole with amplitude $\Delta \simeq 0.006$ (for spectral index $\gamma \simeq 3$ ) due to the Compton-Getting effect. The presence of magnetic fields will however modify the structure of the dipolar anisotropy because the deflections of the particle trajectories lead to a non-trivial mapping between the directions of incidence at the halo and the arrival directions at Earth. The galactic magnetic field, with its regular component, is expected to lead to the largest distortion of the large scale distribution. Turbulent magnetic fields are expected to add some small scale distortion.

Besides the distortion of the anisotropies of the flux reaching the halo due to the magnetic deflections, we describe here also another effect that can lead to anisotropies at the Earth, even if the flux were isotropic outside the halo in the observer's reference frame. This is due to the fact that because of the galactic rotation there is an electric component of the galactic field in the reference frame in which the solar system is at rest. Then, CRs suffer a small change in their momentum as they travel through the Galaxy. This acceleration is a function of the direction, inducing then anisotropies in the flux observed at Earth in a given energy range.

We will analyze here the expected large scale distribution of extragalactic cosmic rays arising from these effects, taking into account the regular galactic magnetic field. A simplified discussion of the expected Compton-Getting effect was presented in ref. [8].

\section{Galactic magnetic field effects on the extragalactic flux}

At energies below the GZK threshold cosmic rays can arrive from cosmological distances and we assume that there is a large number of sources contributing to the CR flux. We will consider that the distribution of this very large number of sources is isotropic in a reference system that may be identified with the CMB rest frame, assuming that the effects of an eventually anisotropic source distribution can be neglected. Due to the solar system motion with respect to that frame, we can model the cosmic ray flux as a dipolar distribution before entering the Galaxy halo.

The presence of the galactic magnetic field along the CR trajectories have two effects on the CR intensity observed at Earth. These arise from the non-trivial correspondence between the arrival direction at Earth and the incidence direction outside the halo due to the magnetic deflections and from a change in magnitude of the particles momentum due to the electric force acting on the particles in the observer frame. We discuss here 
their effects on the flux of particles arriving to the Earth. According to the Liouville theorem [12, 13] the phase space density of cosmic rays is conserved along the particle trajectories. The differential flux of particles, i.e. the number of particles per unit solid angle and unit energy that cross a unit area per unit time, is related to the phase space distribution $f$ by $\Phi(\vec{p}, \vec{r})=p^{2} f(\vec{p}, \vec{r})$. If CRs are only subject to magnetic forces along their trajectories, $p$ is conserved and thus also the intensity of the flux is conserved, but due to the deflections of the particles in the galactic magnetic field, the flux corresponding to a given direction of incidence at the halo will appear at a different direction (or different directions if there are multiple images) as seen from the Earth. On the other hand, due to the high conductivity of the galactic medium, we can consider that there are only magnetic fields present, with no electric fields, in the reference frame moving locally with the plasma. The plasma is known to move approximately with the rotation curve of the Galaxy. Thus, to an observer moving with the solar system, CRs traveling through far away regions of the Galaxy will also experience an electric force coming from the relative motion of the medium in which the field is purely-magnetic, given by $q \vec{E}=(q / c) \Gamma_{\Delta V}\left(\vec{V}-\vec{V}_{\odot}\right) \times \vec{B}^{\prime}$, with $\Gamma_{\Delta V} \simeq 1$ being the Lorentz factor associated to the relative velocity of the local system and the observer, $\Delta \vec{V} \equiv \vec{V}-\vec{V}_{\odot}$, and $\vec{B}^{\prime}$ is the magnetic field in the local frame. The effect on the deflection of the trajectories due to the induced electric field is negligible as compared to the deflection due to the magnetic field, as the relative velocities involved $\vec{V}-\vec{V}_{\odot}$ are at maximum only few hundred $\mathrm{km} / \mathrm{s}$, much smaller than $v$, the velocity of the ultrarelativistic CRs $\left(\vec{V}-\vec{V}_{\odot} \ll v \simeq c\right)$. The equation of motion for particles of charge $q$ in the observer's (solar system) frame can be written as

$$
\dot{\vec{p}} \simeq \frac{q}{c}\left(\left(\vec{V}-\vec{V}_{\odot}\right) \times \vec{B}+\vec{v} \times \vec{B}\right)
$$

where we can consider that $\vec{B} \simeq \vec{B}^{\prime}$ as $\Gamma_{\Delta V} \simeq 1$. The evolution of the direction $\hat{u}$ of the particle $\left(\vec{p}=m \Gamma_{v} v \hat{u}\right)$ is then determined by the second term, $\hat{u}_{f} \simeq \hat{u}_{i}+(q / E) \int \overrightarrow{d l} \times \vec{B}$. However, the change in the absolute value of $\vec{p}$ along the trajectory is determined by the first term:

$$
p_{f}=p_{i}+\frac{q}{c^{2}} \int \overrightarrow{d l} \cdot\left(\left(\vec{V}-\vec{V}_{\odot}\right) \times \vec{B}\right) .
$$

Using the invariance of the phase space distribution along the trajectories (Liouville theorem) we can relate the values at the solar system $f\left(p_{0}, \hat{u}_{0}\right)$ to the value outside the halo $f\left(p_{h}, \hat{u}_{h}\right)$

$$
\begin{aligned}
f\left(p_{0}, \hat{u}_{0}\right) & =f\left(p_{h}, \hat{u}_{h}\right)=f\left(p_{0}, \hat{u}_{h}\right)+\left.\frac{\partial f}{\partial p}\right|_{p_{0}}\left(p_{h}-p_{0}\right) \\
& =f\left(p_{0}, \hat{u}_{h}\right)\left(1+\left.\frac{\partial \ln f}{\partial \ln p}\right|_{p_{0}} \frac{p_{h}-p_{0}}{p_{0}}\right) .
\end{aligned}
$$

Using that $\Phi(p)=f(p) p^{2}$ we can write $\partial \ln f / \partial \ln p=-(\gamma+2)$, where $\gamma$ is the spectral index $\left(\Phi(p) \propto p^{-\gamma}\right)$. Then,

$$
f\left(p_{0}, \hat{u}_{0}\right)=f\left(p_{0}, \hat{u}_{h}\right)\left(1-(\gamma+2) \frac{p_{h}-p_{0}}{p_{0}}\right) .
$$


The phase space distribution outside the halo $f\left(p_{0}, \hat{u}_{h}\right)$ depends on the motion of the solar system with respect to the rest frame where CRs are isotropic, with phase space distribution given by $f^{\prime}\left(p_{0}\right)$. If this coincides with the CMB frame, the relation corresponds to a Compton-Getting dipole with an amplitude $\Delta=(\gamma+2) V_{C M B} / c$ [B],

$$
f\left(p_{0}, \hat{u}_{h}\right)=f^{\prime}\left(p_{0}\right)\left(1+(\gamma+2) \frac{\hat{u}_{h} \cdot \vec{V}_{C M B}}{c}\right) .
$$

Finally, we can relate the flux arriving to the Earth, $\Phi\left(p_{0}, \hat{u}_{0}\right)$, with that outside the halo in the frame where CRs are isotropic, $\Phi^{\prime}\left(p_{0}\right)$, through

$$
\Phi\left(p_{0}, \hat{u}_{0}\right) \simeq \Phi^{\prime}\left(p_{0}\right)\left(1-(\gamma+2) \frac{p_{h}-p_{0}}{p_{0}}+(\gamma+2) \frac{\hat{u}_{h} \cdot \vec{V}_{C M B}}{c}\right) .
$$

The second term in the parenthesis corresponds to the effect of the momentum change along the trajectory, that has to be evaluated through the integral in eq. (2.2).

\section{Quantitative results in a BSS-S model}

Both the mapping between the arrival directions at the halo and the Earth and the change in the momentum of CRs along the trajectory depend on the galactic magnetic field structure and amplitude, that are not well known. As an illustration of the expected effects we will present the results for a regular component of the galactic magnetic field modeled with a bisymmetric field with even symmetry (BSS-S) with structure and strength very similar to those used in ref. [14 but smoothed out as described in ref. [15. In this model the galactic magnetic field reverses its sign between the spiral arms of the Galaxy and the field is symmetric with respect to the Galaxy's mid-plane. The local value of the field is taken as $2 \mu \mathrm{G}$. For the dependence on $z$ a contribution coming from the galactic disk and another one from the halo are considered:

$$
\vec{B}_{r e g}(x, y, z)=\vec{B}_{r e g}(x, y, z=0)\left(\frac{1}{2 \cosh \left(z / z_{1}\right)}+\frac{1}{2 \cosh \left(z / z_{2}\right)}\right)
$$

with $z_{1}=0.3 \mathrm{kpc}$ and $z_{2}=4 \mathrm{kpc}$. We will briefly discuss how the results are modified when some other models are considered. The effects due to the propagation in the galactic magnetic fields on cosmic rays coming from point sources, such as the (de)magnification of the flux, formation of multiple images, or the modification of the spectrum measured at the Earth, have been discussed in refs. [15, 16].

We analyze here the effects of the galactic magnetic fields on a smooth flux distribution on the sky. We first consider the effect of the the momentum change, that is a function of the rotation velocities of the Galaxy, and then the distortion of the Compton-Getting anisotropy due to the magnetic deflection, that is function of the solar system velocity with respect to the CRs rest frame. The trajectories of CRs linking the arrival direction at Earth to the direction of incidence at the halo are obtained by backtracking antiparticles leaving the Earth with a velocity opposite to that of the arriving CR. Performing then the 

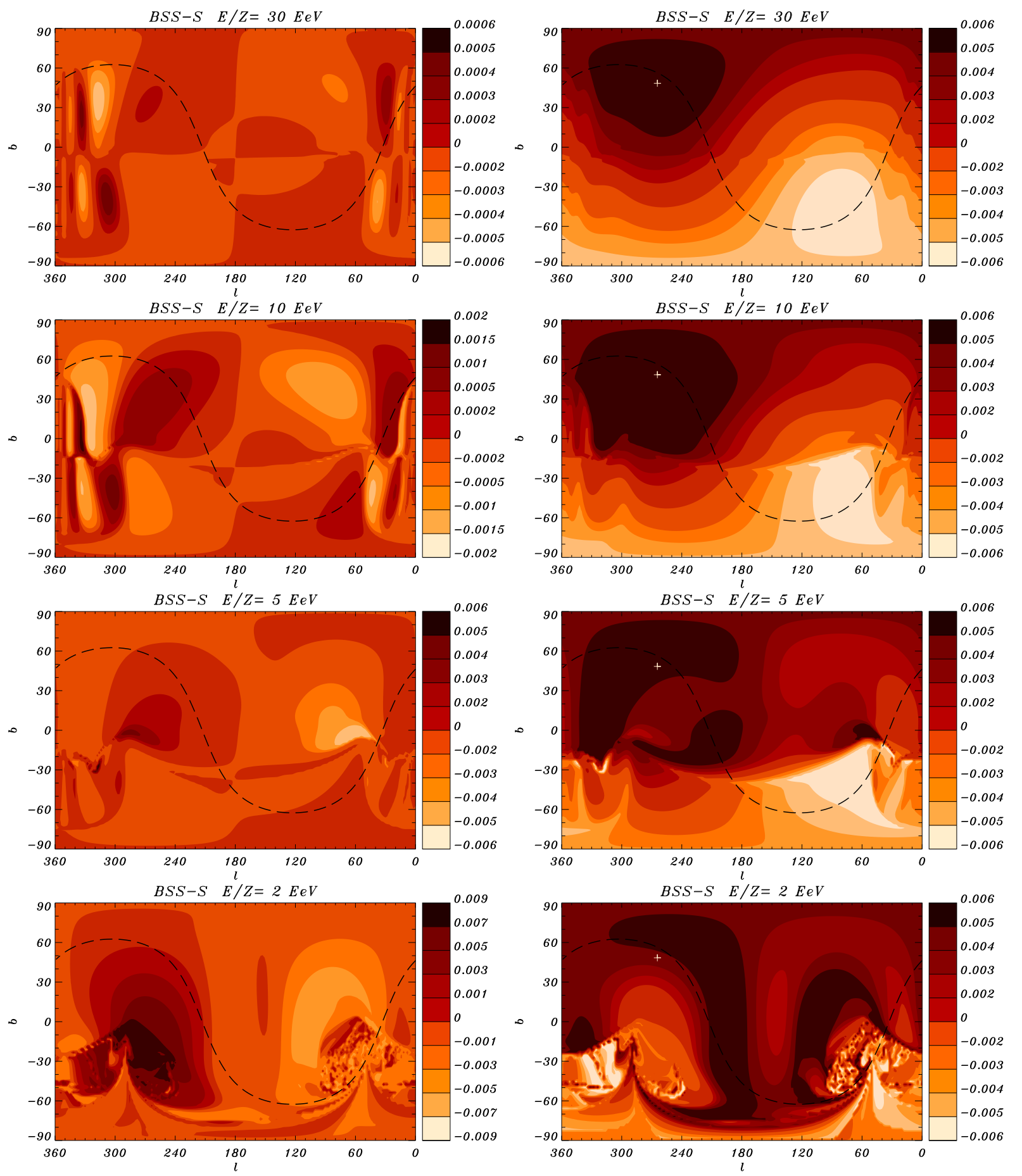

Figure 1: Left panels: modulation of the extragalactic flux caused by the momentum change of CRs due to the electric force along the trajectories for different rigidities. Right panels: ComptonGetting anisotropy distorted by the magnetic deflections for different rigidities.

integral of eq. (2.2) along the trajectories, the change of the momentum as a function of the arrival direction is obtained. We consider that the rest frame of the plasma, in which there 
is only a magnetic field, moves everywhere with a velocity following the rotation curve of the Galaxy, that we approximate as being flat as a function of the distance to the galactic center and with a magnitude of $220 \mathrm{~km} / \mathrm{s}$, in a clockwise direction as seen from the north galactic pole.

We plot in the left panels in Figure 1 the resulting modulation factor on the halo flux considering a spectral index $\gamma=2.7$ and for different values of the CR rigidities $E / Z$ (corresponding to the second term in the right hand side of eq. (2.7)). The galactic magnetic field model considered has only azimuthal and radial components (no component in the direction perpendicular to the galactic plane). As the relative velocities $\vec{V}-\vec{V}_{\odot}$ have also only azimuthal and radial components, the electric force is always perpendicular to the galactic plane, pointing up or down in different spiral arms due to the reversals of the magnetic field. For high rigidities, the trajectories are almost straight, and then for particles traveling in the galactic plane direction the effect is negligible as the electric force is orthogonal to the trajectory and thus does not change the magnitude of the momentum. Also for $l=0^{\circ}$ and $l=180^{\circ}$ the effect is negligible as for those directions the force is very small because the relative velocities and the magnetic field are almost parallel. ${ }^{1}$ The effect has a clear asymmetry with respect to the galactic plane with regions showing an excess of flux in the northern hemisphere accompanied by a region with a flux deficit in the southern hemisphere at the same galactic longitude, and vice-versa. This is due to the fact that an upward pointing electric field at a given longitude accelerate particles arriving from the south, while decelerates particles arriving from the north. For smaller rigidities this pattern becomes distorted because the trajectories are increasingly deflected in the magnetic field. On the other hand, the trajectories inside the halo are longer and the magnitude of the momentum change is larger, leading to an increase of the modulation of the flux, as is evidenced in the change in the color scale of the figures for decreasing rigidities.

The right panels in Figure 1 show the modulation factor on the halo flux due to the Compton-Getting effect if the rest frame where CRs are isotropic coincides with the CMB one, considering a spectral index $\gamma=2.7$ and for different values of the CR rigidities (corresponding to the third term in the right hand side of eq. (2.7)). The direction of the original dipolar component of the extragalactic cosmic ray flux points to the $(b, l)=$ $\left(48.4^{\circ}, 264.4^{\circ}\right)$ direction, indicated by the white cross in the plots and its amplitude is $\Delta \simeq 0.6 \%$. As the rigidity decreases the deflections of the particles grow and the distortions of the dipolar modulation become larger and it is apparent that higher order harmonics become important.

The combined effect of the variation of the momentum along the trajectory and the magnetic deflection distortion of the Compton-Getting dipole is shown in Figure 2 for the same rigidities as in Figure 1.

Figure 3 shows the amplitude of the dipolar component arising from each effect, as well of the total one. For the particular orientation of the dipole considered (CMB rest frame) and the galactic magnetic field model considered, the dipolar amplitude of the Compton-Getting anisotropy is increasingly suppressed as the rigidity decreases below 10

\footnotetext{
${ }^{1}$ These features would change if there is for instance a dipole contribution to the magnetic field, leading to a vertical non-vanishing component, which could be significant in the central regions of the Galaxy.
} 

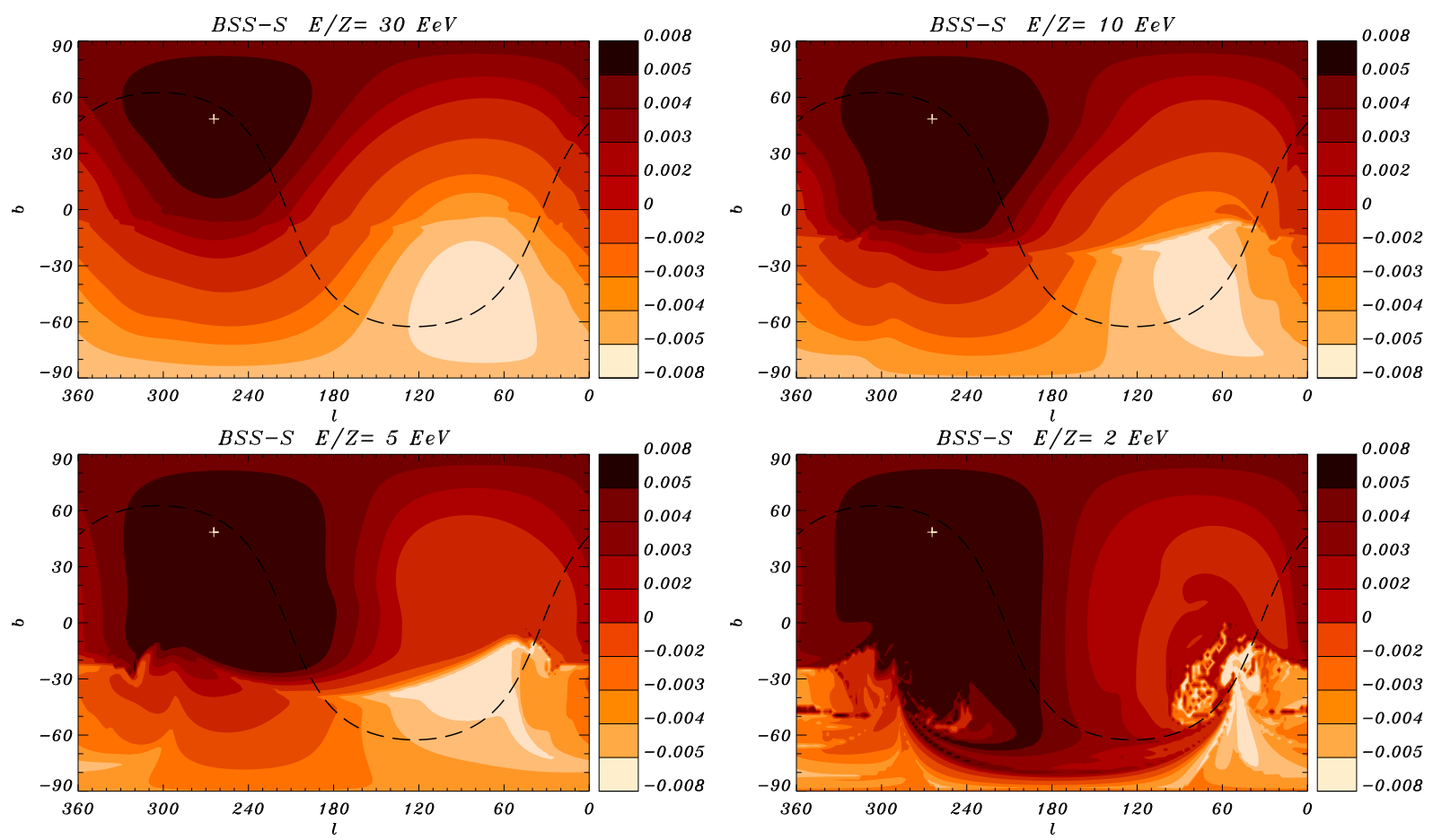

Figure 2: Total modulation of the flux for different rigidities.

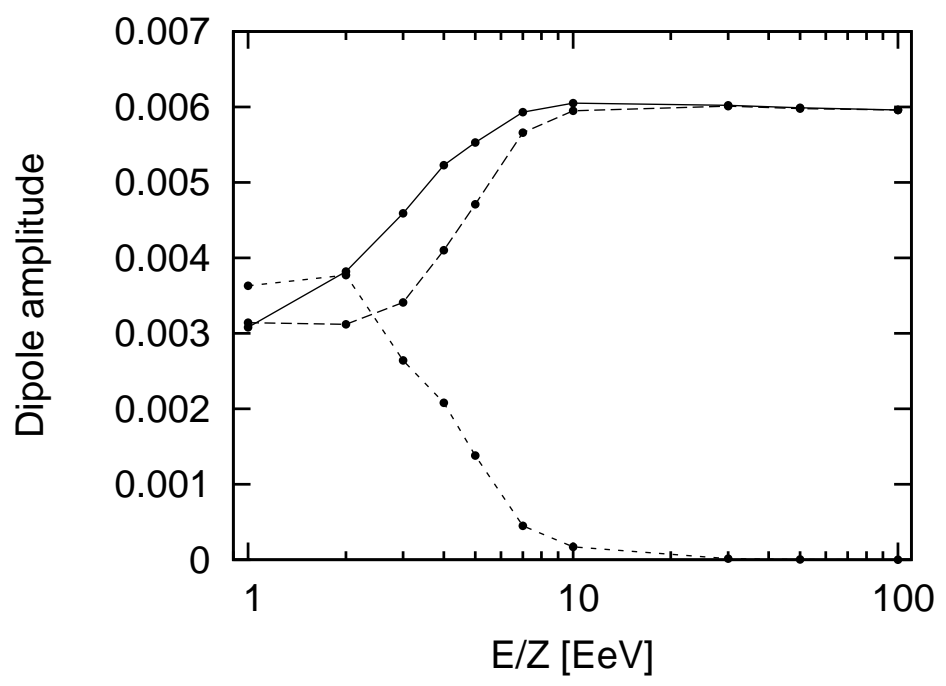

Figure 3: Amplitude of the dipolar component of the flux modulation as a function of the rigidity for the Compton-Getting anisotropy distorted by the magnetic deflections (long-dashed line), for the momentum change induced anisotropy (short-dashed line) and for the sum of both effects (solid line).

$\mathrm{EeV}$, reaching a $50 \%$ suppression for rigidities around $2-3 \mathrm{EeV}$. On the other hand, the anisotropy arising from the momentum variation along the trajectories grows for energies below $10 \mathrm{EeV}$ and around $2-3 \mathrm{EeV}$ its dipolar amplitude is similar to the ComptonGetting one. A curious fact that is evident in the two lower energy panels $(E / Z=5 \mathrm{EeV}$ 
and $2 \mathrm{EeV}$ ) of Figure 1 is that the anisotropy generated through the momentum variation along the trajectories (left panels) have flux excesses (and deficits) located roughly in the same directions where the deficits (and excesses) corresponding to the Compton-Getting anisotropy are located (right panel). As a result of this the total combined anisotropy shown in Figure 2 has a more dipolar-like shape at these lower energies than any of the two individual effects, and the total dipolar amplitude is larger as shown in Figure 3. This is curious because the amplitude of both effects depend on different unrelated velocities. For example, had the Milky Way be moving in the opposite direction with respect to the CMB rest frame, the Compton-Getting modulation of the flux displayed in the right panels in Figure 1 would have had the opposite sign, while the left panels would have been unchanged, and the compensation would not have occurred. These results depend thus on which is the rest frame where CRs are isotropic.

At rigidities below $10 \mathrm{EeV}$ the contribution from higher order harmonics becomes significant as it can be qualitatively appreciated in Figure 1. By expanding the flux reaching the Earth as a series of spherical harmonics with coefficients $a_{\ell m},{ }^{2}$

$$
\Phi(\hat{u})=\sum_{l=0}^{\infty} \sum_{m=-\ell}^{\ell} a_{\ell m} Y_{\ell m}(\hat{u}),
$$

we can quantify the relative amplitude of the different multipoles through the angular power spectrum $C_{\ell}$, that is given by the average over the possible $m$ values of the $a_{\ell m}^{2}$ coefficients,

$$
C_{\ell}=\frac{1}{2 \ell+1} \sum_{m=-\ell}^{\ell} a_{\ell m}^{2} .
$$

We show in Figure 4 the normalized contribution for the three lowest order terms, the dipole, the quadrupole and the octupole as a function of the rigidity. At the highest rigidities only the dipole component is significant, but as the rigidity decreases the quadrupole and octupole components become important.

The previous results have all been obtained for the BSS-S galactic magnetic field model. The main results still hold qualitatively for an ASS-A model (i.e. with no reversals and antisymmetric with respect to the Galactic plane) with the same local amplitude and halo parameters.

The results are relevant for energies such that the extragalactic component of cosmic rays is dominant, that is above $\sim 4 \mathrm{EeV}$ if the ankle marks the galactic to extragalactic transition or at even lower energies in the $e^{+} e^{-}$pair production dip scenario for the ankle. On the other hand, we have assumed that the flux of extragalactic cosmic rays can be approximated as a diffuse dipolar like flux. This approximation holds only for a very large number of sources distributed at cosmological distances. Thus, the energy should be small enough so that the GZK effect does not considerably attenuate the flux from distant sources. For energies below $30 \mathrm{EeV}$ cosmic rays can arrive from distances of about $1 \mathrm{Gpc}$, then this is a safe upper energy limit to consider.

\footnotetext{
${ }^{2}$ we use the real spherical harmonics base, so that the $a_{\ell m}$ are real.
} 


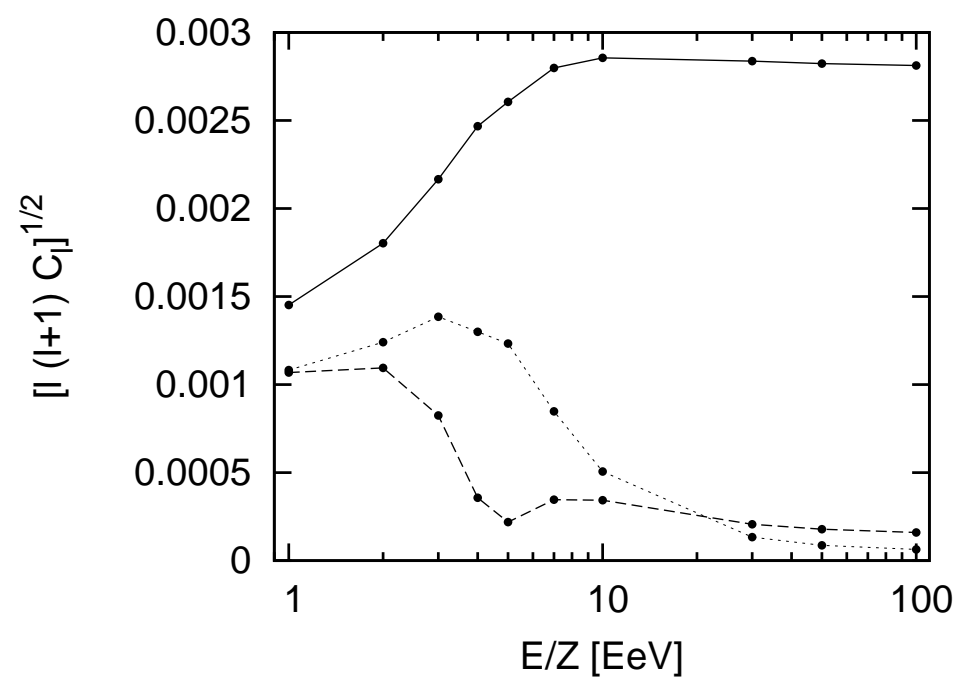

Figure 4: Lowest order power spectrum terms as a function of the rigidity: dipole $(\ell=1$, solid line), quadrupole $(\ell=2$, short dashed line) and octupole $(\ell=3$, long dashed line).

If extragalactic protons are the dominant component of the flux at the ankle energies and below, as in the $e^{+} e^{-}$pair production dip scenario for the ankle, for large enough intergalactic magnetic field values the extragalactic flux may experience a diffusive motion at the lower energies. It has been pointed out that this could lead to the appearance of a magnetic horizon: the time needed for cosmic rays to arrive at Earth from distances larger than this horizon is typically larger than the age of the universe, suppressing the flux arriving from them [17]. This affects the anisotropy of cosmic rays arriving to the halo of the Galaxy as discussed in ref. 18]. If the particles with energies $10^{17}-10^{18} \mathrm{eV}$ arrive predominantly from the closest source through a diffusive propagation, the flux reaching the galactic halo will be dipolar-like with amplitude about $10^{-2}-10^{-3}$. In this case, the propagation of cosmic rays in the galactic magnetic field will also affect the anisotropies observed at Earth, and an analysis along the lines of this work still holds, although the direction of the dipole would not coincide with the CMB one, but would point to the direction to the closest source.

\section{Conclusions}

We have analyzed in detail the impact of the propagation through the galactic magnetic field on the large scale anisotropies of extragalactic cosmic rays. As the propagation is a function of the cosmic ray rigidity, the resulting anisotropy is also a function of the rigidity. On one hand, the Compton-Getting dipolar anisotropy resulting from the motion of the observer with respect to the frame in which the cosmic rays are isotropic is distorted by the magnetic deflection of the trajectories in the galactic magnetic field. In the absence of magnetic field effects, the expected dipole amplitude is of about $0.6 \%$ if the CRs rest frame coincides with the CMB one. The distortion due to the magnetic deflections modifies this amplitude, leading to a suppression by a factor of up to $50 \%$ at energies below $3 \mathrm{EeV}$ for the 
BSS-S galactic magnetic field considered. Moreover, as a consequence of the propagation the general pattern of the flux is distorted and significant higher order harmonics appear in the expected distribution of arrival directions.

On the other hand, even if the observer's rest frame is not moving with respect to the CRs frame and the flux of CRs entering the halo were perfectly isotropic, the flux distribution observed at Earth would be affected by the galactic magnetic field. This is due to the fact that the motion of the galactic medium (and hence of the magnetic field) following the galactic rotation leads to an electric field in the reference frame moving with the solar system. Then, CRs suffer a small change in their momentum as they travel through the Galaxy. This acceleration is a function of the direction, inducing then anisotropies in the flux observed at Earth in a given energy range. This effect grows with decreasing energy, reaching $\sim 0.3 \%$ at energies below $2-3 \mathrm{EeV}$.

Let us mention that in this work we considered the cosmic ray distribution in the whole sky, but for specific experiments on Earth only part of the sky is observed. The details of the results obtained in a given cosmic ray observatory will then depend on the declination dependence of its exposure and hence on the site location. Anyhow the results will be qualitatively similar to those obtained for the full sky. The detection of this effect is a challenge for present experiments. The Pierre Auger Observatory has recently published upper limits for the first harmonic amplitude in this energy range. The 99\% CL upper limit on the amplitude of the equatorial component of a dipole is about $2 \%$ at $1 \mathrm{EeV}$ and about $10 \%$ at $10 \mathrm{EeV}$ [19. If at EeV energies cosmic rays are predominantly extragalactic, with a few fold increase in the present statistics this experiment would become sensitive to a dipole anisotropy of the amplitude discussed here.

\section{Acknowledgments}

This work is supported by ANPCyT (grant PICT2006 13334) and CONICET (grant PIP 01830).

\section{References}

[1] A. H. Compton and I. A. Getting, Phys. Rev. 47, 817 (1935).

[2] D. J. Cutler and D. E. Groom, Nature 322, L434 (1986).

[3] M. Amenomori et al., Phys. Rev. Lett. 93, 061101 (2004).

[4] M. Amenomori et al., Science 314, 439 (2006).

[5] G. Guillian et al., Phys. Rev. D 75, 062003 (2007).

[6] A. Abdo et al., Astrophys. J. 698, 2121 (2009).

[7] M. Aglietta, Astrophys. J. Lett. 692, 130 (2009).

[8] M. Kachelriess and P. D. Serpico, Phys. Lett. B 640, 225 (2006).

[9] C. T. Hill, D. N. Schramm and T. P. Walker, Phys. Rev. D 34, 1622 (1986).

[10] V. Berezinsky, A. Z. Gazizov and S. I. Grigorieva, Phys. Lett. B 612, 147 (2005). 
[11] D. J. Fixsen et al., Astrophys. J. 473,576 (1996).

[12] G. Lemaitre and M. S. Vallarta, Phys. Rev. 43, 87 (1933).

[13] W. F. G. Swann, Phys. Rev. 44, 224 (1935).

[14] T. Stanev, Astrophys. J. 479, 290 (1997).

[15] D. Harari, S. Mollerach and E. Roulet, JHEP 9908, 022 (1999).

[16] D. Harari, S. Mollerach and E. Roulet, JHEP 0002, 035 (2000).

[17] M. Lemoine, Phys. Rev. D 71, 083007 (2005).

[18] R. Aloisio, V. Berezinsky, P. Blasi and S. Ostapchenko, Phys. Rev. D, 77025007 (2008).

[19] R. Bonino, for the Pierre Auger Collaboration, 31st International Cosmic Ray Conference, Lodz, Poland (2009). 